\author{
Deutsche Osteoonkologische Gesellschaft \\ Prof. Dr. med Ingo J. Diel (1. Vorsitzender) \\ Schwerpunktpraxis für gynäkologische Onkologie (SPGO) \\ Quadrat P7, 16-17 \\ 68161 Mannheim, Germany \\ Tel. +49621 12506420 \\ diel@spgo-mannheim.de
}

\title{
Deutsche Osteoonkologische Gesellschaft gegründet
}

Am 24. April 2010 wurde die Deutsche Osteoonkologische Gesellschaft (DOG) neu gegründet. Wie der Gynäkoonkologe und weltweit anerkannte Spezialist für Knochenmetastasen, Prof. Dr. Ingo J. Diel, Mannheim, einführend mitteilte, war die Gründung dieser interdisziplinären Fachgesellschaft längst überfällig, denn tumorbedingte und tumortherapiebedingte Erkrankungen des Skeletts nehmen rasant zu. Bisher stellten jedoch die Erforschung der Ursachen und die Entwicklung von therapeutischen Optionen nur Randgebiete der großen Fachgesellschaften dar. Die wissenschaftliche Fachgesellschaft DOG sieht es daher als ihr primäres Ziel an, osteoonkologisches Wissen innerhalb aller onkologischen Fachdisziplinen zu fördern und zu verbreiten. Insbesondere sollen interdisziplinäre Leitlinien für die Behandlung von Knochenmetastasen und therapiebedingter Osteoporose geschaffen und für deren breite Umsetzung gesorgt werden.

Erster Vorsitzender der DOG ist Prof. Dr. Ingo J. Diel, zum zweiten Vorsitzenden wurde der Orthopäde Prof. Dr. med. Andreas A. Kurth, Mainz gewählt. Dem Vorstand gehören zudem Dr. Holger Uhthoff, Speyer (Schatzmeister) und Prof. Dr. M. Heinrich Seegenschmiedt, Hamburg (Schriftführer) an. Zu Beisitzern wurden PD Dr. Christian Eberhardt, Hanau, Prof. Dr. Tanja Fehm, Tübingen, Prof. Dr. Franz Jakob, Würzburg, und PD Dr. Florian Schütz, Heidelberg berufen.

Die Gesellschaft umfasst derzeit 45 Gründungsmitglieder. Mitglied werden kann jeder, der sich wissenschaftlich mit dem Gebiet der Osteoonkologie beschäftigt. Die Gesellschaft wird als Verein eingetragen und die Gemeinnützigkeit wird beantragt.

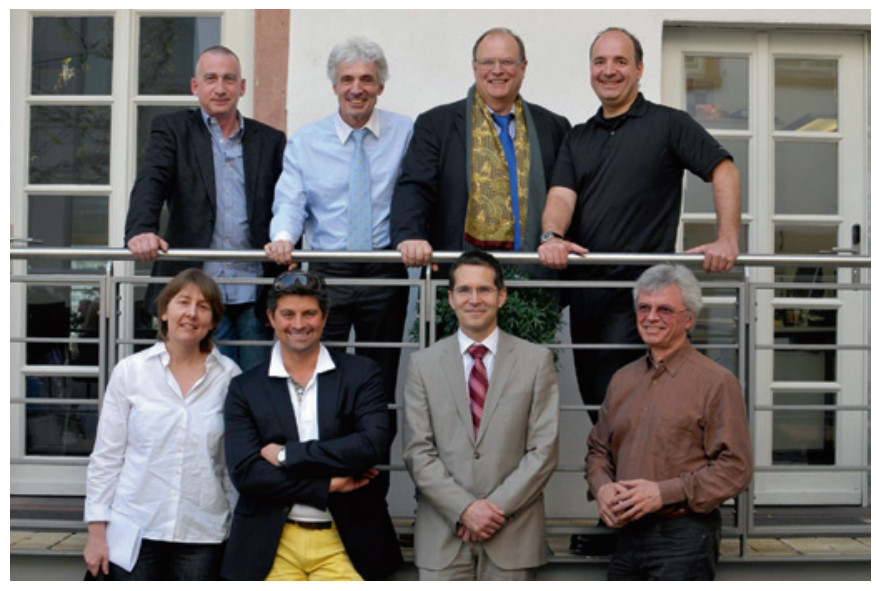

Vorstand der DOG. Von links oben: H. Uhthoff, M.H. Seegenschmiedt, I.J. Diel, A.A. Kurth; unten: T. Fehm, C. Eberhardt, F. Schütz, F. Jakob.

Kontakt und weitere Informationen:

Prof. Dr. med Ingo J. Diel

Schwerpunktpraxis für gynäkologische Onkologie (SPGO)

Quadrat P7, 16-17

68161 Mannheim, Germany

Tel. +4962112506420

diel@spgo-mannheim.de

\section{KARGER}

Fax +49 7614520714

Information@Karger.de

www.karger.com (c) 2010 S. Karger GmbH, Freiburg 